\title{
Multivariate max-stable spatial processes
}

\author{
BY MARC G. GENTON \\ CEMSE Division, King Abdullah University of Science and Technology, Thuwal 23955-6900, \\ Saudi Arabia \\ marc.genton@kaust.edu.sa
}

SIMONE A. PADOAN

Department of Decision Sciences, Bocconi University of Milan, 20136 Milano, Italy

simone.padoan@unibocconi.it

AND HUIYAN SANG

Department of Statistics, Texas A\&M University, College Station, Texas 77843, U.S.A. huiyan@stat.tamu.edu

\section{SUMMARY}

Max-stable processes allow the spatial dependence of extremes to be modelled and quantified, so they are widely adopted in applications. For a better understanding of extremes, it may be useful to study several variables simultaneously. To this end, we study the maxima of independent replicates of multivariate processes, both in the Gaussian and Student- $t$ cases. We define a Poisson process construction and introduce multivariate versions of the Smith Gaussian extreme-value, the Schlather extremal-Gaussian and extremal- $t$, and the Brown-Resnick models. We develop inference for the models based on composite likelihoods. We present results of Monte Carlo simulations and of an application to daily maximum wind speed and wind gust.

Some key words: Composite likelihood; Cross-correlation; Extremal coefficient; Max-stable process; Multivariate analysis; Random field; Spatial extreme.

\section{INTRODUCTION}

The statistical modeling of spatial extremes of natural processes is important in environmental studies to understand the probability of events such as floods, heat waves, or hurricanes. Extreme events in space can be described by max-stable processes (de Haan \& Ferreira, 2006), which extend the generalized extreme-value distribution. In a seminal unpublished University of Surrey 1990 technical report, R. L. Smith, using the de Haan (1984) spectral representation, proposed a spatial max-stable model, named the Gaussian extreme-value process, whose dependence structure is obtained using Gaussian densities. Schlather (2002) extended the de Haan formulation to random functions and proposed a model, named the extremal-Gaussian process. Another popular spatial max-stable model is the Brown-Resnick process (e.g., Kabluchko et al., 2009). The extremal-Gaussian max-stable model based on compact random sets was introduced by Schlather (2002) and used by Davison \& Gholamrezaee (2012). Spatio-temporal max-stable processes have been discussed by Kabluchko (2009), Davis et al. (2013) and Huser \& Davison (2014). Asymptotically independent processes for modelling extreme values were discussed by de Haan \& Zhou (2011), Wadsworth \& Tawn (2012) and Padoan (2013a). Reviews of recent 
advances in the statistical modeling of spatial extremes are Davison et al. (2012), Cooley et al. (2012), Padoan (2013b), Ribatet (2013) and Davison \& Huser (2014).

Although the aforementioned literature attests to vigorous research, it is limited to modeling a single variable. In practice, however, multiple variables are often observed. For instance, rainfall, temperature and wind may be observed at many locations spread across a region. Each of these variables has its own spatial variation but also depends on the other variables. The aim of this paper is to extend the theory and application of max-stable processes to the multivariate setting.

45 Zhang \& Smith (2004) investigated the behavior of multivariate maxima of moving maxima processes, but these are not suitable for modeling spatial extremes. There is thus a need to extend max-stable processes to the multivariate setting and to make them practically useful.

To fix notation, let $I=\{1, \ldots, p\}$ and $K=\{1, \ldots, q\}$ be sets of indices of variables and of spatial locations, respectively. Let $N=p q, J=I \times K$ be the Cartesian product and $J_{i k}=$ $\{(j, l) \in J \backslash(i, k)\}$. We denote by $\left\{Y_{i}(s)\right\}_{s \in \mathcal{S}}$ a real-valued random process on $\mathcal{S} \subseteq \mathbb{R}^{d}$, with $i \in$ $\mathbb{N}$. Let $Y(s)=\left\{Y_{1}(s), \ldots, Y_{p}(s)\right\}^{\mathrm{T}}$ be a $p$-vector of processes observed in $\mathcal{S}$ and $\left\{Y^{(m)}(s)\right\}_{m \geq 1}$ be independent copies of $Y(s)$.

\section{MAXIMA OF INDEPENDENT REPLICATES OF MULTIVARIATE PROCESSES}

\subsection{Gaussian case}

For every $n \in \mathbb{N}$, let $\left\{Y_{n}(s)\right\}_{s \in \mathcal{S}}$ be a $p$-dimensional Gaussian process that is second-order stationary with a zero-mean vector function, unit variances, and a matrix-valued covariance function, $\Sigma(h ; n)=\left\{\rho_{i j}(h ; n)\right\}_{i, j \in I}, h \in \mathbb{R}^{d}$. Specifically, $\rho_{i j}(h ; n)=E\left\{Y_{i n}(s) Y_{j n}(s+h)\right\}$ is the spatial cross-correlation function between processes $i$ and $j$, with $i \neq j$, and $h$ is the spatial lag between locations. When $j=i, \rho_{i i}(h ; n)$ is the correlation function of process $i$. If $h=0$, then $\rho_{i i}=1$ and $\rho_{i j}$ represents the correlation between components. For simplicity of notation, we write $\rho_{i j}(n)$ and $\rho_{i}(h ; n)$ for $\rho_{i j}(0 ; n)$ and $\rho_{i i}(h ; n)$, respectively.

Let $b_{n}$ be a sequence such that $(2 \pi)^{1 / 2} b_{n} \exp \left(b_{n}^{2} / 2\right) \sim n$ as $n \rightarrow \infty$ (Resnick, 1987, Ch. 1).

Assumption 1. Suppose that the dependence structure, $\Sigma(h ; n)$, of $Y_{n}(s)$ depends on $n$ with

$$
2 b_{n}^{2}\{\Upsilon-\Sigma(h ; n)\} \rightarrow \Lambda(h), \quad n \rightarrow \infty,
$$

where $\Upsilon$ is a $p \times p$ matrix of ones and $\Lambda(h)$ is a matrix-valued non-negative function. By this, we mean that, for the correlation structure, the following constraints are fulfilled: ${ }_{65} 2 b_{n}^{2}\left\{1-\rho_{i j}(h ; n)\right\} \rightarrow \lambda_{i j}^{2}(h)$ as $n \rightarrow \infty$, for all $h \in \mathbb{R}^{d}$ and for any $i, j \in I$, where $\lambda_{i j}^{2}(h)$ is a non-negative function. When $j \neq i$ and $h=0,2 b_{n}^{2}\left\{1-\rho_{i j}(n)\right\} \rightarrow \lambda_{i j}^{2}$, as $n \rightarrow \infty$, where $\lambda_{i j}^{2} \in[0, \infty)$ is assumed. When $j=i$ and $h=0$, then $\rho_{i}(n)=1$ and $\lambda_{i}^{2}=0$.

The quantity $\lambda_{i j}^{2}$ measures the strength of the dependence among the variables in the limit, $\lambda_{i}^{2}(h)$ and $\lambda_{i j}^{2}(h)$ represent the limiting spatial inter-component and cross-component dependence functions. If Assumption 1 holds, then we derive the following generalization of the Hüsler-Reiss model (Hüsler \& Reiss, 1989; Kabluchko et al., 2009; Kabluchko, 2011).

Proposition 1. For every $n \in \mathbb{N}$, let $\left\{Y_{n}^{(m)}(s)\right\}_{m=1}^{n}$ be independent copies of $Y_{n}(s)$ and let $M_{n}(s)=\left\{M_{i n}(s)\right\}_{i \in I}$ be the vector of pointwise maxima, where $M_{i n}(s)=$ $\max _{m=1, \ldots, n}\left\{Y_{i n}^{(m)}(s)\right\}$, for all $s \in \mathcal{S}$. Take $Z_{n}(s)=b_{n}\left\{M_{n}(s)-b_{n}\right\}$. Then, $Z_{n}(s) \rightarrow Z(s)$ 75 weakly as $n \rightarrow \infty$ and $\{Z(s)\}_{s \in \mathcal{S}}$ is a p-dimensional max-stable process with a finite dimensional distribution, for positive values $\left\{z_{i}\left(s_{k}\right)\right\}_{(i, k) \in J}$ with $s_{k} \in \mathcal{S}$, equal to 
$\exp \left(-V_{1 \cdots p}^{[N]}\left[\left\{z_{i}\left(s_{k}\right)\right\}_{(i, k) \in J}\right]\right)$ where $N=p q$ and $V_{1 \cdots p}^{[N]}\left[\left\{z_{i}\left(s_{k}\right)\right\}_{(i, k) \in J}\right]$ equals

$$
\sum_{(i, k) \in J} \frac{1}{z_{i}\left(s_{k}\right)} \Phi_{N-1, \bar{\Lambda}_{i k}}\left(\left[\frac{\lambda_{i j}\left(s_{k}-s_{l}\right)}{2}+\frac{\log \left\{z_{j}\left(s_{l}\right) / z_{i}\left(s_{k}\right)\right\}}{\lambda_{i j}\left(s_{k}-s_{l}\right)}\right]_{(j, l) \in J_{i k}}\right),
$$

where $\Phi_{N-1, \bar{\Lambda}_{i k}}$ is the $(N-1)$-dimensional Gaussian distribution function with mean zero and partial correlation matrix, $\bar{\Lambda}_{i k}$, provided that this matrix is invertible.

The proof and the form of $\bar{\Lambda}_{i k}$ are reported in the Supplementary Material. Here, $V_{1 \cdots p}^{[N]}\left[\left\{z_{i}\left(s_{k}\right)\right\}_{(i, k) \in J}\right]$ is a multivariate exponent function; see de Haan \& Ferreira (2006, Ch. 9) for a discussion of the univariate case. The superscript on $V$ means that it is an exponent function of $N$ random components, that is, $p$ variables observed at $q$ sites. The subscript lists the variables involved. The overall dependence among the $p$ spatial variables is fully described by (1), which depends on the spatial cross-component dependence, $\lambda_{i j}\left(s_{k}-s_{l}\right)$, the individual spatial dependence, $\lambda_{i}\left(s_{k}-s_{l}\right)$, and the dependence between variables, $\lambda_{i j}$. We call the process $Z(s)$ with exponent function (1) the multivariate Hüsler-Reiss process. In (1), if we set a common threshold, $z$, then the multivariate extremal coefficient is

$$
\theta_{1 \cdots p}^{[N]}\left(\left\{s_{k}-s_{l}\right\}_{k, l \in K}\right)=\sum_{(i, k) \in J} \Phi_{N-1, \bar{\Lambda}_{i k}}\left[\left\{\lambda_{i j}\left(s_{k}-s_{l}\right) / 2\right\}_{(j, l) \in J_{i k}}\right] .
$$

In this case, $1 \leq \theta_{1 \cdots p}^{[N]}\left(\left\{s_{k}-s_{l}\right\}_{k, l \in K}\right) \leq N$, where the lower bound represents the complete dependence case, arising when all variables are totally dependent, whereas the upper bound represents independence (Schlather \& Tawn, 2003).

Since for a large number of variables and locations, $\theta_{1 \ldots p}^{[N]}\left(\left\{s_{k}-s_{l}\right\}_{k, l \in K}\right)$ may be difficult to compute, interpret and represent graphically, we propose to use the following lower-order extremal coefficients to summarize the dependence of multiple spatial extremes. Consider a pair of variables, $\left(Z_{i}, Z_{j}\right), i, j \in I(i \neq j)$, observed at two sites separated by $h \in \mathbb{R}^{d}$. A natural summary of the spatial dependence between the extremes of two variables is the pairwise crosscomponent extremal coefficient, $\theta_{i j}^{[2]}(h)=2 \Phi\left\{\lambda_{i j}(h) / 2\right\} \in[1,2]$. In general, $\theta_{i j}^{[2]}(h) \neq \theta_{j i}^{[2]}(h)$, since $\lambda_{i j}(h) \neq \lambda_{j i}(h)$, because $\rho_{i j}(h) \neq \rho_{j i}(h)$. For a full understanding of the dependence, $\theta_{i j}^{[2]}(h), \theta_{j i}^{[2]}(h), \theta_{i}^{[2]}(h), \theta_{j}^{[2]}(h)$ and $\theta_{j i}^{[2]}$ should be considered together. A richer measure that simultaneously uses the information of $\lambda_{i j}(h), \lambda_{j i}(h), \lambda_{i}(h), \lambda_{j}(h)$ and $\lambda_{i j}$ is the quadruplewise spatial extremal cross-component coefficient,

$$
\begin{aligned}
\theta_{i j}^{[4]}(h)= & \Phi_{3, \bar{\Lambda}_{1}}\left\{\frac{\lambda_{i}(h)}{2}, \frac{\lambda_{i j}}{2}, \frac{\lambda_{i j}(h)}{2}\right\}+\Phi_{3, \bar{\Lambda}_{2}}\left\{\frac{\lambda_{i}(h)}{2}, \frac{\lambda_{j i}(h)}{2}, \frac{\lambda_{i j}}{2}\right\} \\
& +\Phi_{3, \bar{\Lambda}_{3}}\left\{\frac{\lambda_{j}(h)}{2}, \frac{\lambda_{i j}}{2}, \frac{\lambda_{j i}(h)}{2}\right\}+\Phi_{3, \bar{\Lambda}_{4}}\left\{\frac{\lambda_{j}(h)}{2}, \frac{\lambda_{i j}(h)}{2}, \frac{\lambda_{i j}}{2}\right\} \in[1,4] .
\end{aligned}
$$

This is derived from the distribution of $\left\{Z_{i}(s), Z_{j}(s), Z_{i}(s+h), Z_{j}(s+h)\right\}$, provided in the Supplementary Material with the matrices, $\bar{\Lambda}_{i}(i=1, \ldots, 4)$. The coefficient in (2) satisfies $\theta_{i j}^{[4]}(h)=\theta_{j i}^{[4]}(h)$, and the pairwise coefficients, $\theta_{i j}^{[2]}(h), \theta_{j i}^{[2]}(h), \theta_{i}^{[2]}(h), \theta_{j}^{[2]}(h)$ and $\theta_{i j}^{[2]}$ are spe- 95 cial cases. 
We can also consider $\left(Z_{i}, Z_{j}, Z_{v}\right)$ with $i, j, v \in I(i \neq j \neq v)$, separated by $h, h^{\prime}, h^{\prime \prime} \in \mathbb{R}^{d}$, giving the triplewise spatial extremal cross-component coefficient,

$$
\begin{aligned}
\theta_{i j v}^{[3]}\left(h, h^{\prime}, h^{\prime \prime}\right)= & \Phi_{2, \bar{\Lambda}_{1}}\left\{\frac{\lambda_{i j}(h)}{2}, \frac{\lambda_{i v}\left(h^{\prime}\right)}{2}\right\}+\Phi_{2, \bar{\Lambda}_{2}}\left\{\frac{\lambda_{i j}(h)}{2}, \frac{\lambda_{j v}\left(h^{\prime \prime}\right)}{2}\right\} \\
& +\Phi_{2, \bar{\Lambda}_{3}}\left\{\frac{\lambda_{i v}\left(h^{\prime}\right)}{2}, \frac{\lambda_{j v}\left(h^{\prime \prime}\right)}{2}\right\} \in[1,3] .
\end{aligned}
$$

This summarizes the extremal dependence for $\left\{Z_{i}(s), Z_{j}(s+h), Z_{v}\left(s+h^{\prime}\right)\right\}$. Although $\theta_{i j v}^{[3]}\left(h, h^{\prime}, h^{\prime \prime}\right)$ is not invariant with respect to the order of the variables, it represents a compromise between complexity and interpretability.

\subsection{Correlation models for the Gaussian case}

The validity of result (1) depends on whether Assumption 1 is met. Such constraints are satisfied by the class of multivariate Gaussian random fields with sufficiently smooth spatial isotropic cross-correlation functions for which, around $h=0$,

$$
\rho_{i j}(h ; n)=1-c_{n} \lambda_{i j}^{2}-c_{n} \psi\|h\|^{\kappa}+o\left(c_{n}^{2}\right),
$$

for a sequence of constants, $c_{n}$, such that $c_{n} \rightarrow 0$ as $n \rightarrow \infty$, where $0<\kappa \leq 2$ is a smoothness parameter, and $\psi$ and $\lambda_{i j}^{2}, i, j \in I$, are non-negative constants. An example of a multivariate correlation model satisfying (4) is the class of power exponential correlation

$$
\rho(h) \equiv \rho(h ; \alpha, \kappa)=\exp \left\{-\left(\frac{\|h\|}{\alpha}\right)^{\kappa}\right\} \quad(\alpha>0,0<\kappa \leq 2),
$$

where the parameters $\kappa$ and $\alpha$ represent the smoothness and the scale of the spatial random field. The cross-correlation functions, with any $i, j \in I$ and $j \neq i$, are defined by $\rho_{i j}(h)=\rho_{j i}(h)=$ $\rho_{i j} \rho(h)$, which contains a factor $\rho_{i j}$ that expresses the correlation between the variables. Considering a correlation matrix, $\Sigma=\left\{\rho_{i j}\right\}_{i, j \in I}$, among components, the overall dependence, $\Sigma(h)$, emerges as a non-negative definite correlation matrix. This correlation model is obtained as a product between the inter- and cross-correlations. It is therefore separable between the variables and the spatial domain. It includes the exponential correlation, $\kappa=1$, and the Gaussian correlation, $\kappa=2$.

Define $\rho_{i j}(h ; n)=\rho_{i j}(n) \rho\left(c_{n}^{\kappa} h\right)$, where $\rho_{i j}(n)=1-c_{n} \lambda_{i j}^{2}+o\left(c_{n}\right)$ and $\rho\left(c_{n}^{\kappa} h\right)=1-$ $c_{n} \psi\|h\|^{\kappa}+o\left(c_{n}\right)$, for $c_{n} \rightarrow 0$ as $n \rightarrow \infty$. Then, choosing $c_{n}=\left(2 b_{n}^{2}\right)^{-1}$, it follows that $2 b_{n}^{2}\left\{1-\rho_{i j}(h ; n)\right\} \rightarrow \lambda_{i j}^{2}(h)$, where $\lambda_{i j}^{2}(h)=\lambda_{i j}^{2}+\lambda^{2}(h)$ is the spatial cross-component dependence function, with $\lambda_{i j}$ the cross-component parameter and $\lambda^{2}(h)=(\|h\| / \alpha)^{\kappa}$ the spatial dependence function. With this correlation model, $\psi=\alpha^{-\kappa}$ in (4) and $\rho_{i j}(h)=\rho_{j i}(h)$ so that $\theta_{i j}^{[2]}(h)=\theta_{j i}^{[2]}(h)$, thus (2) simplifies to

$$
\theta_{i j}^{[4]}(h)=4 \Phi_{3, \bar{\Lambda}_{1}}\left\{\lambda(h) / 2, \lambda_{i j} / 2, \lambda_{i j}(h) / 2\right\} .
$$

Although $\rho_{i j}(h)$ is separable, the dependence structure of the limiting distribution is not. Indeed, (6) is not a product of two coefficients, one describing the spatial dependence and the other the dependence among variables. Further properties of (6) are reported next. Set $h=\|h\|$. When the variables are completely dependent, that is, $\lambda_{i j}=0$, then $\lambda_{i j}(h)=\lambda(h)$ and hence $\theta_{i j}^{[4]}(0)=1$ and $\theta_{i j}^{[4]}(h) \rightarrow 2$ as $h \rightarrow \infty$. Thus, (6) behaves as the usual pairwise spatial coefficient. When the variables are dependent, that is, $0<\lambda_{i j}<\infty$, then $1<\theta_{i j}^{[4]}(h)<4$ for any $h>0$ and it is 

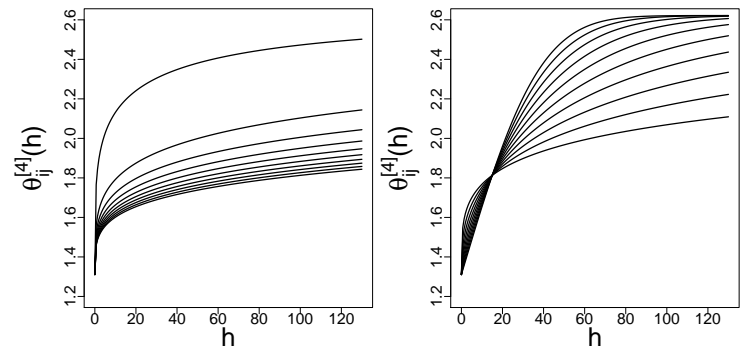

Fig. 1. Quadruplewise extremal coefficient functions of the bivariate Hüsler-Reiss process with power exponential cross-correlation. Left panel: the curves from top to bottom correspond to 10 equally spaced values of $\alpha$ from 1 to 100 and fixed $\kappa=0.5$; right panel: the curves from bottom to top correspond to 10 equally spaced values of $\kappa$ from 0.5 to 2 and fixed $\alpha=15$. In both cases, $\lambda_{i j}=0.8$ and $h=\|h\|$.
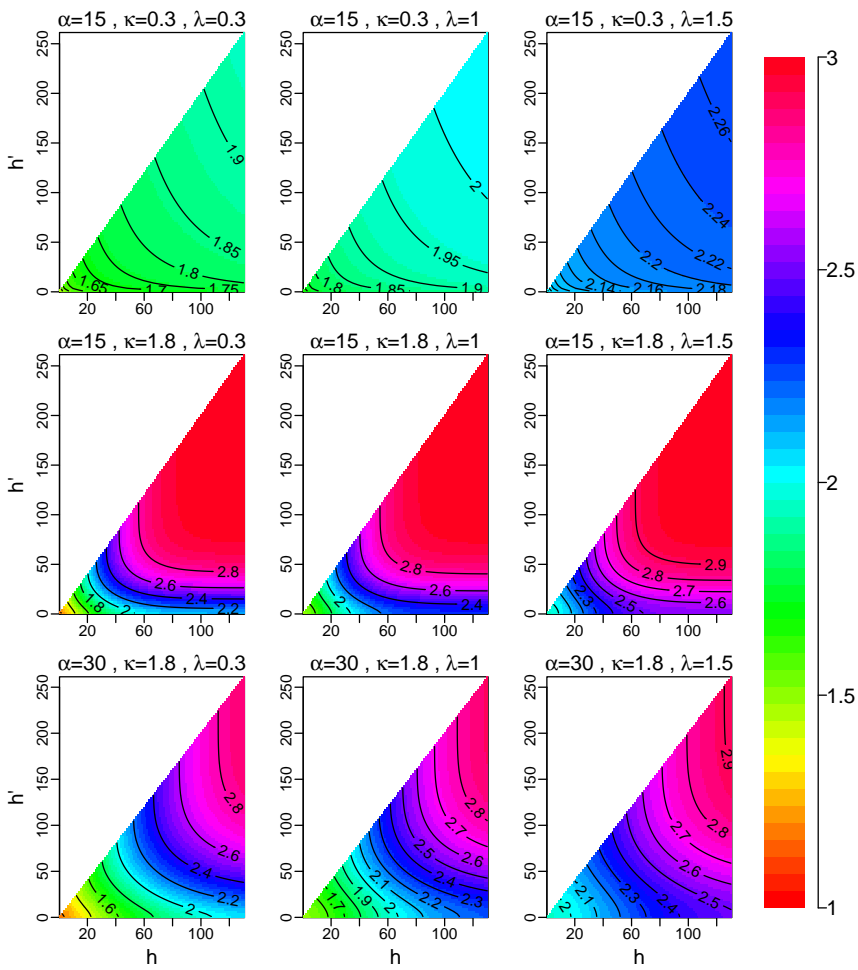

Fig. 2. Triplewise extremal coefficient functions of the trivariate Hüsler-Reiss process with power exponential cross-correlation. Reported is $\theta_{i j v}^{[3]}\left(h, h, h^{\prime}\right)$ with $h=\|h\|$, $h^{\prime} \in(0,2 h]$, different parameter values and $\lambda_{i j}=\lambda_{i v}=$ $\lambda_{j v}=\lambda$. Extremal coefficients with short, medium and long range are reported from the top to the bottom panels.

$1<\theta_{i j}^{[4]}(0)<2$ if $h=0$. Thus, (6) behaves like the usual pairwise cross-component coefficient.

When the variables are independent, that is, $\lambda_{i j}=\infty$, then $\theta_{i j}^{[4]}(0)=2$ if $h=0$ and $\theta_{i j}^{[4]}(h)=4$ 


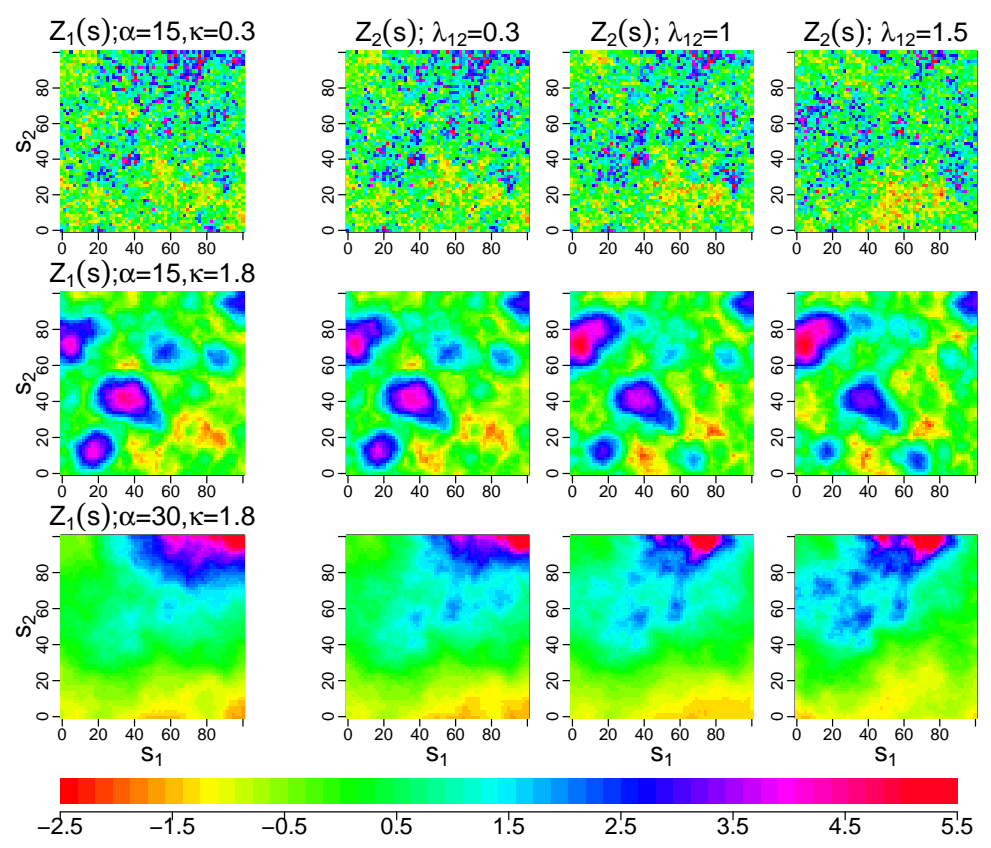

Fig. 3. Simulated bivariate Hüsler-Reiss max-stable random fields having a power exponential cross-correlation function at a mesh grid of $60 \times 60$ on $[0,100]^{2}$. Margins are on the standard Gumbel scale.

for any $h>0$. Figure 1 depicts the behavior of (6). When $h=0$, the coefficient shows the dependence between the two variables and ignores the spatial component. This can be interpreted as an average dependence between variables in the area. More detailed information is available when $h>0$. We see that the spatial dependence between variables decreases with distance, increases with increasing scale for a fixed smoothness, and increases/decreases at long/short distances with increasing smoothness at a fixed scale. Figure 2 depicts the triplewise coefficient function (3) when $h^{\prime}=h$ and $h^{\prime \prime} \in(0,2 h]$, using a power exponential cross-correlation function. Depending on the parameter configuration, the extremal coefficient function exhibits short-, mediumor long-range variation. Figure 3 plots simulated bivariate Hüsler-Reiss random fields having a power exponential correlation function. A more flexible cross-correlation model would allow for different scale parameters, $\alpha_{i j}$, and smoothing parameters, $\kappa_{i j}(i, j \in I)$. Unfortunately, a characterization of the values of its parameters that leads to a valid cross-correlation model does not appear to be known (Genton \& Kleiber, 2015). Another flexible cross-correlation model is the multivariate Matérn covariance function (Gneiting et al., 2010; Apanasovich et al., 2012), but, this cannot be used to derive (1) since even in the univariate case it does not satisfy the required condition (4).

\subsection{Student-t case}

Similarly to the univariate case (Røislien \& Omre, 2006), we say that $\{Y(s)\}_{s \in \mathcal{S}}$ is a $p$ dimensional Student- $t$ process with vector centering and matrix scale-dependence functions, $\left\{\mu_{i}(s)\right\}_{i \in I}$ and $\Sigma(s)=\left\{\sigma_{i j} \rho_{i j}(s)\right\}_{i, j \in I}$, and $\nu>0$ degrees of freedom, if for all possible sequences, $\left\{s_{k}\right\}_{k \in K} \in \mathcal{S},\left\{Y\left(s_{k}\right)\right\}_{k \in K}$ has an $N$-dimensional Student- $t$ distribution, $N=p q$, with a vector of centers, $\left\{\mu_{i}\left(s_{k}\right)\right\}_{(i, k) \in J}$, a positive definite $N \times N$ scale-dependence matrix, 
$\Sigma=\left\{\sigma_{i j} \rho_{i j}\left(s_{k}-s_{l}\right)\right\}_{(i, k),(j, l) \in J}$, and $\nu$ degrees of freedom. Without loss of generality, in the sequel, we assume that $\mu_{i}(s)=0$ and $\sigma_{i j}=1$ for all $i, j \in I$, such that the correlation function and the degrees of freedom determine the dependence structure of the multivariate Student- $t$ random field. Then, the following result can be derived.

Let $\left\{Y^{(m)}(s)\right\}_{m=1, \ldots, n}$ be a sequence of independent and identically distributed copies of $Y(s)$ and let $M_{n}(s)=\left\{M_{i n}(s)\right\}_{i \in I}$ be the vector of pointwise maxima. Consider the normalized vector of pointwise maxima, $Z_{n}(s)=M_{n}(s) / a_{n}(s)$, where $a_{n}(s)>0$ is an appropriate vector of normalizing functions. Then, $Z_{n}(s) \rightarrow Z(s)$ weakly as $n \rightarrow \infty$, where $Z(s)$ is a $p$ dimensional max-stable process with a finite dimensional distribution that depends on the exponent function

$$
\sum_{(i, k) \in J} \frac{1}{z_{i}\left(s_{k}\right)} T_{N-1, \bar{\Sigma}_{i k}, \nu+1}\left\{\left(\left\{\frac{\nu+1}{1-\rho_{i j}^{2}\left(s_{k}-s_{l}\right)}\right\}^{1 / 2}\left[\left\{\frac{z_{j}\left(s_{l}\right)}{z_{i}\left(s_{k}\right)}\right\}^{1 / \nu}-\rho_{i j}\left(s_{k}-s_{l}\right)\right]\right)_{(j, l) \in J_{i k}}\right\},
$$

where $T_{N-1, \bar{\Sigma}_{i k}, \nu+1}$ is an $(N-1)$-dimensional Student- $t$ cumulative distribution function with zero centers, partial correlation matrix $\bar{\Sigma}_{i, k}$, and $\nu+1$ degrees of freedom; see the Supplementary Material for details. We call the process $Z(s)$ with distribution (7) the multivariate extremal$t$ process; for the univariate case, see Nikoloulopoulos et al. (2009), Davison et al. (2012), and Opitz (2013). In this case, the spatial extremal cross-component coefficient is

$\theta_{1 \cdots p}^{[N]}\left(\left\{s_{k}-s_{l}\right\}_{k, l \in K}\right)=\sum_{(i, k) \in J} T_{N-1, \bar{\Sigma}_{i, k}, \nu+1}\left\{\left(\left[\frac{(\nu+1)\left\{1-\rho_{i j}\left(s_{k}-s_{l}\right)\right\}}{1+\rho_{i j}\left(s_{k}-s_{l}\right)}\right]^{1 / 2}\right)_{(j, l) \in J_{i k}}\right\}$.

Triplewise and quadruplewise extremal coefficient functions similar to those in (2) and (3) can be derived. The pairwise spatial extremal cross-component coefficient is

$$
\theta_{i j}^{[2]}(h)=2 T_{\nu+1}\left(\left[(\nu+1)\left\{1-\rho_{i j}(h)\right\} /\left\{1+\rho_{i j}(h)\right\}\right]^{1 / 2}\right),
$$

where $T_{\nu+1}$ is a univariate Student- $t$ cumulative distribution function with $\nu+1$ degrees of freedom, $h \in \mathbb{R}^{d}$. The benefit of working with the multivariate extremal- $t$ process rather than with the Hüsler-Reiss model is that we can use the multivariate Matern covariance function (Gneiting et al., 2010; Apanasovich et al., 2012). Nonseparable cross-correlation models can be constructed using latent dimensions as proposed by Apanasovich \& Genton (2010). Asymmetric cross-correlation models ( $\mathrm{Li} \&$ Zhang, 2011) and spatially varying cross-correlation coefficients (Kleiber \& Genton, 2013) could be investigated as well; see Genton \& Kleiber (2015) for a review of cross-covariance models for multivariate random fields.

\section{A POISSON POINT PROCESS CONSTRUCTION}

\subsection{Multivariate extension}

Let $X(s)=\left\{X_{1}(s), \ldots, X_{p}(s)\right\}^{\mathrm{T}}$ be a real-valued $p$-dimensional random function on $\mathcal{S}$ and let $X^{(m)}, m \geq 1$, be independent and identically distributed copies of it. Define, for all $s \in \mathcal{S}$, $W(s)=\left\{\max \left\{X_{1}(s), 0\right\}, \ldots, \max \left\{X_{p}(s), 0\right\}\right\}^{\mathrm{T}}$. Assume that, for all $s \in \mathcal{S}$ and $i \in I$,

$$
E\left\{W_{i}(s)\right\}=\tau \in(0, \infty), \quad E\left\{\sup _{s \in \mathcal{S}} W_{i}(s)\right\}<\infty .
$$


Let $\left\{R^{(m)}\right\}_{m>1}$ be points of an inhomogeneous Poisson process on $\mathbb{R}_{+}$with intensity measure $d r / r^{2}$. If the $R^{(m)}$ 's are independent of the $W^{(m)}$ 's, then

$$
Z(s)=\max _{m=1,2, \ldots}\left\{R^{(m)} W^{(m)}(s)\right\} / \tau \quad(s \in \mathcal{S})
$$

defines a $p$-dimensional max-stable process on $\mathcal{S}$ with unit Fréchet margins, where the maximum is taken componentwise. If $p=1$, then we obtain the univariate spectral representation of max-stable processes (de Haan, 1984; Schlather, 2002; de Haan \& Ferreira, 2006, Ch. 9). For a finite set of spatial points, $\left\{s_{k}\right\}_{k \in K} \in \mathcal{S}$, and positive thresholds, $\left\{z_{i}\left(s_{k}\right)\right\}_{i \in I}$, for any given $k \in K$, with similar arguments as in Schlather (2002) and Ch. 9 in de Haan \& Ferreira (2006), the finite dimensional distribution is $\operatorname{pr}\left\{Z_{i}\left(s_{k}\right) \leq z_{i}\left(s_{k}\right),(i, k) \in J\right\}=$ $\exp \left(-V_{1 \cdots p}^{[N]}\left[\left\{z_{i}\left(s_{k}\right)\right\}_{(i, k) \in J}\right]\right)$, where the exponent function is

$$
V_{1 \cdots p}^{[N]}\left[\left\{z_{i}\left(s_{k}\right)\right\}_{(i, k) \in J}\right]=\tau^{-1} E\left[\max _{(i, k) \in J}\left\{W_{i}\left(s_{k}\right) / z_{i}\left(s_{k}\right)\right\}\right] .
$$

The process (9) is max-stable since its finite-dimensional distributions are max-stable (de Haan, 1984). The exponent function in (10) is a spatial cross-component exponent function which expresses the dependence among $p$ variables observed at $q$ spatial points. Since (10) is a homogenous function of order -1 , the overall spatial cross-component extremal coefficient is

$$
\theta_{1 \cdots p}^{[N]}\left(\left\{s_{k}-s_{l}\right\}_{k, l \in K}\right)=\tau^{-1} E\left\{\max _{(i, k) \in J} W_{i}\left(s_{k}\right)\right\} \in[1, N] .
$$

A special case of $(10)$ is

$$
V_{i j}^{[2]}\left\{z_{i}(s), z_{j}(s+h)\right\}=\tau^{-1} E\left[\max \left\{W_{i}(s) / z_{i}(s), W_{j}(s+h) / z_{j}(s+h)\right\}\right] .
$$

This is expressed as a function of the separation vector, $h$, so that the pairwise spatial crosscomponent extremal coefficient is $\theta_{i j}^{[2]}(h)=V_{i j}^{[2]}(1,1)$, which is equal to $\theta_{i}^{[2]}(h)$ when $j=i$ and to $\theta_{i j}^{[2]}$ when $h=0$. Finally, given $p$ variables observed at $q$ locations, from the exponent function (10), we can deduce that:

a) $\theta_{1 \cdots p}^{[N]}\left(\left\{s_{k}-s_{l}\right\}_{k, l \in K}\right)=\theta_{i_{1} \cdots i_{p}}^{[N]}\left(\left\{s_{k}-s_{l}\right\}_{k, l \in K}\right)$ for any $i_{1}, \ldots, i_{p} \in I$;

b) $\theta_{1 \cdots p}^{[N]}\left(\left\{s_{k}-s_{l}\right\}_{k, l \in K}\right)=\theta_{1 \ldots p}^{[N]}\left(\left\{s_{l}-s_{k}\right\}_{k, l \in K}\right)$;

c) for every $v=2, \ldots, p, \theta_{1 \cdots v}^{[v]}\left(\left\{s_{k}-s_{l}\right\}_{k, l \in 1, \ldots, v}\right) \neq \theta_{i_{1} \cdots i_{v}}^{[v]}\left(\left\{s_{k}-s_{l}\right\}_{k, l \in 1, \ldots, v}\right)$, for any $i_{1} \neq$ $\cdots \neq i_{v} \in I$, and $\theta_{1 \cdots v}^{[v]}\left(\left\{s_{k}-s_{l}\right\}_{k, l \in 1, \ldots, v}\right) \neq \theta_{1 \cdots v}^{[v]}\left(\left\{s_{l}-s_{k}\right\}_{k, l \in 1, \ldots, v}\right)$.

Thus $\theta_{1 \cdots p}^{[N]}\left(\left\{s_{k}-s_{l}\right\}_{k, l \in K}\right)$ provides a richer summary than $\theta_{1 \cdots v}^{[v]}\left(\left\{s_{k}-s_{l}\right\}_{k, l \in 1, \ldots, v}\right)$, with $v=$ $2, \ldots, p$.

\subsection{Multivariate max-stable spatial models}

Different choices for $W$ allow us to derive different multivariate models for spatial extremes.

In (9), define $W(s)=\exp \left\{X(s)-\sigma^{2}(s) / 2\right\}$, where $X(s)$ is a zero-mean, $p$-dimensional Gaussian process with stationary increments and a finite vector of variances, $\sigma^{2}(s)=$ $\left\{\sigma_{i}^{2}(s)\right\}_{i \in I}$, where $\sigma_{i}^{2}(s)=\operatorname{var}\left\{X_{i}(s)\right\}$, and assume that $X$ has a matrix-valued variogram, $2 \gamma(h)=\left\{2 \gamma_{i j}(h)\right\}_{i, j \in I}$, where $\gamma_{i j}: \mathcal{S} \rightarrow[0, \infty)$, with $\gamma_{i j}(0)=0$, is a conditionally negative definite function, called the semi-variogram. Specifically, $2 \gamma_{i j}(h)=\operatorname{var}\left\{X_{i}(s+h)-X_{j}(s)\right\}$ 
is the spatial cross-variogram between the processes $i$ and $j(i \neq j)$. By definition of the variogram, we have

$$
2 \gamma_{i j}(h)=\sigma_{i}^{2}(s+h)+\sigma_{j}^{2}(s)-2 \operatorname{cov}\left\{X_{i}(s+h), X_{j}(s)\right\} \quad(i, j \in I) .
$$

When $i=j, 2 \gamma_{i}(h)=\operatorname{var}\left\{X_{i}(s+h)-X_{i}(s)\right\}$ is the purely spatial inter-variogram function for component $i$ and, finally, when $h=0$, we obtain $2 \gamma_{i j}=\operatorname{var}\left\{X_{i}(s)-X_{j}(s)\right\}$. In this case, the constant in (8) is $\tau=1$ and the resulting process, $Z$, is named the multivariate Brown-Resnick model. The finite dimensional distribution of $Z$ has an exponent function equal to (1) but where $\lambda_{i j}\left(s_{k}-s_{l}\right)=\left\{2 \gamma_{i j}\left(s_{k}-s_{l}\right)\right\}^{1 / 2}$ and the partial correlation matrix $\bar{\Lambda}_{i k}$ depends on the crossvariograms, $2 \gamma_{i j}\left(s_{k}-s_{l}\right)$, for $(i, k),(j, l) \in J$. This result is obtained following the argument of Huser \& Davison (2013) (see also Wadsworth \& Tawn, 2014); see the Supplementary Material for details. The pairwise cross-component extremal coefficient is $\theta_{i j}^{[2]}(h)=2 \Phi\left[\left\{\gamma_{i j}(h) / 2\right\}^{1 / 2}\right]$. In general, the cross-variogram can be asymmetric, that is, $2 \gamma_{i j}(h) \neq 2 \gamma_{j i}(h)$. If it is symmetric, then the spatial cross-component extremal coefficient simplifies further. In particular, if the multivariate Gaussian process, $X(s)$, has a spatial cross-variogram, $2 \gamma_{i j}(h)=\lambda_{i j}^{2}+\lambda_{i}^{2}(h)$, where $\lambda_{i j}^{2} \in[0, \infty)$ and $2 \gamma_{i j}(h)=\lambda_{i j}^{2}+\|h / \alpha\|^{\kappa}(\alpha>0, \kappa \in(0,2])$, we obtain the same dependence structure as in $\S 2.3$. Finally, if $X(s)$ is a second-order, zero-mean, stationary, $p$-dimensional Gaussian process with $\sigma(s)=\{\sigma, \ldots, \sigma\}^{\mathrm{T}}$ for all $s \in \mathcal{S}$, then $Z$ is the multivariate version of the geometric Gaussian process proposed by Davison et al. (2012), whose exponent function is equal to (1) but where $\lambda_{i j}\left(s_{k}-s_{l}\right)=\left[2 \sigma\left\{1-\rho_{i j}\left(s_{k}-s_{l}\right)\right\}\right]^{1 / 2}$.

In (9), define $W(s)=\max \{0, X(s)\}^{\nu}$, where $X(s)$ is a zero-mean, unit-variance, $p$ dimensional Gaussian process with matrix-valued covariance function $\Sigma(h)=\left\{\rho_{i j}(h)\right\}_{i, j \in I}$ and $\nu>0$. In this case, the constant in (8) is $\tau=2^{(\nu-2) / 2} \Gamma\{(\nu+1) / 2\} / \sqrt{ } \pi$ and the resulting process is named the multivariate extremal- $t$. The finite-dimensional distribution of $Z$ has exponent function equal to (7) (Opitz, 2013). A positive aspect when working with the multivariate extremal- $t$ process is that we can use flexible cross-correlation functions, $\rho_{i j}(h)$, such as the multivariate Matérn (Gneiting et al., 2010; Apanasovich et al., 2012) that can have different spatial scales and smoothnesses for the variables, or other models (Genton \& Kleiber, 2015) that, for example, allow us to control the nonseparability between space and variables.

In (9), define $W(s)=\left\{f_{1}\left(X^{(m)}-s\right), \ldots, f_{p}\left(X^{(m)}-s\right)\right\}$, where $f_{i}, i \in I$, is a unimodal continuous probability density on $\mathbb{R}^{d},\left\{X^{(m)}\right\}_{m \geq 1}$ are points of a homogeneous Poisson process on $\mathbb{R}^{d}$ with positive intensity measure $\delta(d x)$. If $f$ is a Gaussian density then the process, $Z$, provides the multivariate version of the Gaussian extreme-value process due to R. L. Smith and mentioned in $\S 1$. Results in the Supplementary Material suggest that, in contrast to the univariate case, the bivariate spatial distribution of the Gaussian extreme-value process is different from that of the Hüsler-Reiss or Brown-Resnick processes.

\section{INFERENCE}

Composite likelihood inference (e.g., Varin et al., 2011) is well-tested with univariate maxstable processes (Padoan et al., 2010; Davison \& Gholamrezaee, 2012; Ribatet et al., 2012). Denote by $\vartheta$ the vector of all the unknown model parameters of a multivariate max-stable process. Here we develop three marginal composite likelihood functions that are useful for parameter estimation in the multivariate case. Suppose that $\left\{z_{i}\left(s_{k}\right)^{(t)}, i \in I, k \in K\right\}_{t=1}^{T}$, with $T \in \mathbb{N}$, are independent and identically distributed realisations from one of the $p$-dimensional max-stable processes described in $\S 2$ and $\S 3$ at a finite set of $q$ locations. For simplicity, we assume that $\mathcal{S} \subset$ $\mathbb{R}^{2}$. Since the model parameters, $\vartheta$, can be identified from the marginal densities, they can be 
estimated by maximizing

$$
\ell_{2}-\operatorname{ci}(\vartheta)=\sum_{t=1, \ldots, T} \sum_{(i, j, l, k) \in D_{2}} w_{i j k l} \log f\left\{z_{i}\left(s_{k}\right)^{(t)}, z_{j}\left(s_{l}\right)^{(t)} ; \vartheta\right\},
$$

where

$$
D_{2}=\left\{(i, j, l, k): \begin{array}{ll}
l>k, k \in K, & j=i, i \in I, \\
l, k \in K, & j>i, i \in I,
\end{array}\right.
$$

$f(\cdot, \cdot ; \vartheta)$ is the density of a pairwise marginal distribution of a multivariate max-stable process, and $\left\{w_{i j k l}:(i, j, l, k) \in D_{2}\right\}$ is a set of nonnegative weights. The subscript ci in (11) stands for cross-inter pairs. Indeed, the log pairwise-likelihood involves all the possible distinct bivariate densities corresponding to any of the following: pairs of variables, spatial pairs of individual variables and spatial pairs of the crossed variables. If $I$ is composed of a single element, e.g., $I=\{1\}$, then (11) turns out to be the log pairwise-likelihood of a univariate max-stable process. When the trivariate marginal distribution of a multivariate max-stable process can also be derived, one can consider the triplewise-likelihood function

$$
\ell_{3}-\operatorname{ci}(\vartheta)=\sum_{t=1, \ldots, T} \sum_{(i, j, v, l, k, r) \in D_{3}} w_{i j v k l r} \log f\left\{z_{i}\left(s_{k}\right)^{(t)}, z_{j}\left(s_{l}\right)^{(t)}, z_{v}\left(s_{r}\right)^{(t)} ; \vartheta\right\},
$$

where

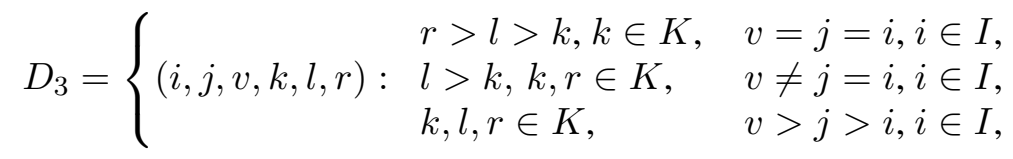

and $\left\{w_{i j v k l r}:(i j v k l r) \in D_{3}\right\}$ are weights. However, given $p$ variables observed at $q$ locations, the number of all possible distinct pairs and triples is $(p q) ! /\{(p q-m) ! m !\}$ with $m=2,3$. Thus, for large $p q$, which is the case for most multivariate spatial applications, to estimate $\vartheta$, numerically maximizing the pairwise and triplewise composite-likelihoods might be too computationally demanding. Therefore, in order to combine a smaller number of triplewise likelihoods, we propose to weight (12) so as to obtain the weighted triplewise likelihood

$$
\begin{aligned}
\ell_{3}-\mathrm{w}(\vartheta)= & \sum_{t=1, \ldots, T} \sum_{j>i \in I, l>k \in K}\left[\log f\left\{z_{i}\left(s_{k}\right)^{(t)}, z_{i}\left(s_{l}\right)^{(t)}, z_{j}\left(s_{k}\right)^{(t)} ; \vartheta\right\}\right. \\
& +\log f\left\{z_{i}\left(s_{k}\right)^{(t)}, z_{i}\left(s_{l}\right)^{(t)}, z_{j}\left(s_{l}\right)^{(t)} ; \vartheta\right\}+\log f\left\{z_{i}\left(s_{k}\right)^{(t)}, z_{j}\left(s_{k}\right)^{(t)}, z_{j}\left(s_{l}\right)^{(t)} ; \vartheta\right\} \\
& \left.+\log f\left\{z_{i}\left(s_{l}\right)^{(t)}, z_{j}\left(s_{k}\right)^{(t)}, z_{j}\left(s_{l}\right)^{(t)} ; \vartheta\right\}\right] .
\end{aligned}
$$

In (13), each single likelihood contribution is formed by all the triplewise marginal likelihoods obtainable from combining two variables where one of the two is observed at two locations. In this way, the spatial cross-dependence and inter-dependence structures are always evaluated in each term. The benefit of working with (13) is that the associated composite likelihood estimators have smaller variances than those of (11)-(12). Each evaluation of (13) involves $p q(p-1)(q-1)$ terms, fewer than the pairwise and triplewise likelihoods. For the latter likelihoods, one can consider the marginal likelihoods corresponding to only the spatial pairs or triples between the crossed variables to decrease the computational cost. This means that the inner sums in the likelihoods (11) and (12) are restricted to the index sets $D_{2}^{*}=\{(i, j, l, k): l, k \in K$ and $j>i, i \in I\}$ and $D_{3}^{*}=\{(i, j, v, l, k, r): k, l, r \in K$ and $v>j>i, i \in I\}$. In these cases, the number of terms involved in each likelihood evaluation is $p ! p ! q ! /\{(p-m) !(q-m) ! m ! m !\}$ respectively 
with $m=2,3$. An alternative method (Sang \& Genton, 2014) is based on tapering of the composite likelihood.

\section{Monte Carlo StUdy}

We simulated $T=30$ independent realizations of a bivariate Hüsler-Reiss process at 35 random locations uniformly in a square of side $[0,100]$ and computed the estimates of the range parameter, $\hat{\alpha}$, the smoothness parameter, $\hat{\kappa}$, and the cross-coefficient, $\hat{\lambda}_{12}$. The simulations were repeated 1000 times to compute the empirical mean squared errors of the parameter estimates. For each simulated data set, we estimated the model parameters using the multivariate extremalcoefficient-based and F-madogram-based approaches (Cooley et al., 2006), and the composite likelihood approach. For the multivariate extremal-coefficient- and $F$-madogram-based approaches, we consider all possible pairs, while, for the composite likelihood approach, we consider (11), which takes into account all possible pairs, the pairwise likelihood version that takes into account only crossed pairs between different variables and locations, and (13).

The estimation methods provide essentially unbiased estimators for the configuration of parameters that we considered. Results are reported in the Supplementary Material. Table 1 shows that some methods are more efficient than others. The $F$-madogram estimator performed better than the extremal-coefficient estimator in almost all parameter settings. Overall, these two empirical estimators performed better than the pairwise likelihood estimator using only crossvariable-different-location pairs. The pairwise likelihood estimators that use all pairs outperform the empirical estimators. Although the pairwise likelihood based only on crossed pairs provides fairly quick estimates, a considerable loss of information is to be expected. In almost all cases, the relative global efficiency of the pairwise likelihood based on all the pairs, relative to triplewise weighted likelihood, is less than 1 , with the smallest value being $64 \%$. The same is true for the relative global efficiencies. This indicates an efficiency gain from using our proposed likelihood over all the other methods. In addition, the benefit of using the weighted triplewise

Table 1. Relative efficiency (\%) relative to the weighted triplewise likelihood estimator. For different parameter values, $\alpha, \kappa$ and $\lambda_{12}$ (reported in brackets) and estimators based on the extremal-coefficient $\left(\theta\right.$-ci), the $F$-madogram $\left(\nu_{F}-c i\right)$ and the pairwise likelihood $\left(\ell_{2}\right.$-ci) using all pairs and the pairwise likelihood using cross-variable-different-location $\left(\ell_{2}-c\right)$, the relative efficiencies $\left(R E_{\alpha}, R E_{\kappa}\right.$ and $\left.R E_{\lambda}\right)$ and the relative global efficiency $(R G E)$ are reported.

\begin{tabular}{|c|c|c|c|c|c|c|c|c|c|c|c|c|c|c|}
\hline $\begin{array}{c}(30,0.5,0.3) \\
\theta-\mathrm{ci}\end{array}$ & $\begin{array}{c}\mathrm{RE}_{\alpha} \\
66\end{array}$ & $\mathrm{RE}_{\kappa}$ & $\begin{array}{c}\mathrm{RE}_{\lambda} \\
65\end{array}$ & RGE & $(30,0.3,0.8)$ & $\mathrm{RE}_{\alpha}$ & $\mathrm{RE}_{\kappa}$ & $\mathrm{RE}_{\lambda}$ & RGE & $(5,0.3,0.3)$ & $\mathrm{RE}_{\alpha}$ & $\mathrm{RE}_{\kappa}$ & $\mathrm{RE}_{\lambda}$ & $\begin{array}{c}\text { RGE } \\
20\end{array}$ \\
\hline$\nu_{F}$-ci & 83 & 61 & 100 & 73 & & 43 & 69 & 78 & 45 & & 45 & 77 & 100 & 66 \\
\hline$\ell_{2}-\mathrm{c}$ & 88 & 14 & 3 & 11 & & 36 & 3 & 17 & 5 & & 6 & 8 & 1 & 2 \\
\hline$\ell_{2}$-ci & 97 & 93 & 94 & 93 & & 97 & 87 & 64 & 74 & & 96 & 100 & 100 & 100 \\
\hline$(30,0.5,0.8)$ & & & & & $(30,1.8,0.8)$ & & & & & $(5,1,0.8)$ & & & & \\
\hline$\theta-\mathrm{ci}$ & 77 & 16 & 60 & 26 & & 68 & 11 & 60 & 17 & & 17 & 14 & 52 & 21 \\
\hline$\nu_{F}$-ci & 87 & 57 & 76 & 61 & & 94 & 33 & 85 & 46 & & 49 & 55 & 90 & 71 \\
\hline$\ell_{2}-\mathrm{c}$ & 62 & 6 & 16 & 11 & & 80 & 20 & 82 & 30 & & 10 & 14 & 3 & 6 \\
\hline$\ell_{2}$-ci & 94 & 80 & 65 & 70 & & 95 & 74 & 83 & 77 & & 84 & 80 & 91 & 82 \\
\hline$(30,0.5,1.5)$ & & & & & $(30,1.8,1.5)$ & & & & & $(15,1,0.8)$ & & & & \\
\hline$\theta$-ci & 73 & 16 & 50 & 22 & & 72 & 11 & 46 & 15 & & 49 & 13 & 54 & 22 \\
\hline$\nu_{F}$-ci & 83 & 65 & 63 & 60 & & 94 & 33 & 60 & 40 & & 91 & 42 & 79 & 55 \\
\hline$\ell_{2}-\mathrm{c}$ & 34 & 3 & 72 & 10 & & 27 & 10 & 96 & 14 & & 44 & 13 & 21 & 15 \\
\hline$\ell_{2}$-ci & 98 & 88 & 53 & 67 & & 95 & 74 & 59 & 64 & & 98 & 75 & 74 & 73 \\
\hline
\end{tabular}


likelihood is more pronounced when the spatial dependence range is at a larger scale or when the cross-dependence is weak. Finally, we also notice that the efficiency gain of using the weighted triplewise likelihood is not always more substantial for smoother spatial fields, as found in Genton et al. (2011) and Huser \& Davison (2013). This suggests that in the multivariate case, our proposed composite likelihood can be beneficial from the point of view of statistical and computational efficiency.

A second simulation study was performed considering a trivariate Hüsler-Reiss max-stable process. The results corroborate in higher dimension the findings above and are reported in the Supplementary Material.

\section{WIND DATA}

We illustrate our method using the monthly maximum of daily maximum wind speed and the monthly maximum of daily maximum wind gust, in miles per hour, at 83 monitoring stations in Oklahoma, U.S.A. The state of Oklahoma has abundant wind resources with high potential capacity for wind power generation. Investigation of occurrence of extreme wind plays a central role in better understanding, predicting, and managing the risks posed by high wind speeds. We derived the monthly maximum wind data from March to May each year from 1994-2008, the windiest season in Oklahoma, from daily observations obtained from the Oklahoma Mesonet database. Our goal is to investigate the spatial extremal dependence structure within and between the extremes of wind speed and gust.

For simplicity, we first separately transformed each variable at each location to a unit Fréchet distribution. We considered three bivariate max-stable process models: the Hüsler-Reiss model with a bivariate power exponential correlation and the extremal-Gaussian and $t$ models, both with a bivariate parsimonious Matérn correlation (Gneiting et al., 2010), which allows two different smoothnesses but equal spatial scales. For comparison with the univariate case, we estimated the dependence parameters by maximizing the pairwise likelihood (11). Confidence intervals for the maximum pairwise likelihood estimates were calculated using the bootstrap. In order to compare the multivariate max-stable processes, we calculated the scaled composite likelihood information criterion (Davison \& Gholamrezaee, 2012) as CLIC ${ }^{*}=-2 c\left[\ell_{2}(\hat{\vartheta})-\operatorname{tr}\left\{\hat{\mathcal{J}}(\hat{\vartheta}) \hat{\mathcal{H}}(\hat{\vartheta})^{-1}\right\}\right]$, where $\ell_{2}(\hat{\vartheta})$ is the pairwise likelihood computed at the maximum, $\hat{\mathcal{J}}$ and $\hat{\mathcal{H}}$ are estimates of $\mathcal{J}(\vartheta)=$ $\operatorname{var}\left\{\nabla \ell_{2}(\vartheta)\right\}$ and $\mathcal{H}(\vartheta)=E\left\{-\nabla^{2} \ell_{2}(\vartheta)\right\}$, and $c$ is chosen such that the log pairwise likelihood is comparable to the log pairwise likelihood under independence. The univariate processes were fitted to the extremes of wind speed and gust separately. CLIC* values were obtained for each univariate process by calculating the pairwise likelihood based on all cross-inter pairs while assuming spatial independence between two variables. In this way, the CLIC* values among univariate and bivariate processes are comparable.

Table 2 reports the estimation results. The results from the univariate models indicate that the range and the smoothness parameters are not significantly different between the wind speed and gust fields. This supports the use of a power exponential correlation with a constant range and smoothness in the bivariate Hüsler-Reiss random field, and a parsimonious bivariate Matérn with a constant range in the bivariate extremal-Gaussian and extremal- $t$ fields. The estimated cross-correlations from the bivariate processes indicate strong correlation between wind speed and gust. The estimated smoothness parameter from the bivariate extremal- $t$ models is slightly smaller than those from the Hüsler-Reiss and bivariate extremal-Gaussian models, and the smoothness of the wind gust field is similar to that of the wind speed. Overall, the smoothness parameters of the bivariate process are small, suggesting rough random fields. According to $\mathrm{CLIC}^{*}$, all three multivariate models fit the data better than their univariate counterparts. The 
Table 2. Estimates of the extremal dependence parameters, their $95 \%$ bootstrap confidence intervals and CLIC* scores under each max-stable process. The scale parameter, $\alpha$, is expressed in kilometers. In the univariate case, estimates concerning monthly maxima of wind speed (WS) and wind gust $(W G)$ are reported for each model. In the bivariate case, estimates of $\kappa$ relate to wind speed and gust maxima, for the extremal-Gaussian and t processes. $\lambda_{12}$ and $\rho_{12}$ are the cross-component dependence parameters for the bivariate Hüsler-Reiss and extremal-t processes. $\nu$ is the degrees of freedom of the extremal-t process.

\begin{tabular}{|c|c|c|c|c|c|c|}
\hline \multicolumn{7}{|c|}{ Univariate random fields } \\
\hline Parameters & & $\alpha$ & $\kappa$ & & $\nu$ & CLIC $^{*}$ \\
\hline \multicolumn{7}{|l|}{ Models } \\
\hline Hüsler-Reiss & WS & $1.7(0.5,6.3)$ & $0.44(0.35,0.62)$ & & & 70712 \\
\hline- & WG & $2.6(1.5,5.9)$ & $0.50(0.40,0.66)$ & & & - \\
\hline Extremal-Gaussian & WS & $28(29,49)$ & $0.49(0.27,0.55)$ & & & 63170 \\
\hline- & WG & $39(26,47)$ & $0.41(0.43,0.72)$ & & & - \\
\hline Extremal- $t$ & WS & $119(99,214)$ & $0.31(0.22,0.41)$ & & $1.75(1.48,2.68)$ & 63092 \\
\hline- & WG & $108(100,155)$ & $0.34(0.26,0.43)$ & & $2.09(1.43,2.96)$ & - \\
\hline \multicolumn{7}{|c|}{ Bivariate random fields } \\
\hline Parameters & & $\alpha$ & $\kappa$ & $\lambda_{12} / \rho_{12}$ & $\nu$ & CLIC $^{*}$ \\
\hline \multicolumn{7}{|l|}{ Models } \\
\hline Hüsler-Reiss & & $2.8(0.9,7.6)$ & $0.49(0.38,0.66)$ & $0.95(0.87,1.04)$ & & 64791 \\
\hline Extremal-Gaussian & WS & $29(15,53)$ & $0.58(0.41,0.83)$ & $0.76(0.73,0.78)$ & & 62345 \\
\hline- & WG & - & $0.46(0.27,0.73)$ & - & & - \\
\hline Extremal- $t$ & WS & $114(100,172)$ & $0.38(0.29,0.50)$ & $0.87(0.84,0.90)$ & $1.98(1.53,2.82)$ & 62251 \\
\hline- & WG & - & $0.30(0.21,0.45)$ & - & - & - \\
\hline
\end{tabular}

bivariate extremal- $t$ model has the smallest CLIC ${ }^{*}$ among all three multivariate models, followed by the bivariate extremal-Gaussian model, suggesting that a multivariate correlation model that allows for different smoothnesses is preferred to one with a single smoothness parameter. The extremal practical ranges (Davison et al., 2012) were calculated from the bivariate extremal- $t$ model. Wind speed has practical ranges $(6.9,107) \mathrm{km}$, which are longer than $(3.5,88) \mathrm{km}$, the practical ranges of wind gust. The extremal cross-component coefficient, $\theta_{i j}^{[2]}$, has an estimated lower bound of 1.32 . The estimate of $\theta_{i j}^{[2]}$ reaches 1.7 beyond 274 kilometers.

Figure 4 plots the binned and unbinned empirical estimates of extremal coefficients against the distance, obtained with the $F$-madogram and the fitted extremal coefficient functions, obtained with a pairwise-likelihood estimator under each bivariate random field.

The fitted inter- and cross-component extremal coefficient functions, obtained with the extremal- $t$ random field, match the binned empirical estimates well. The estimates obtained with the bivariate Hüsler-Reiss random field appear to underestimate the extremal dependence to some extent, perhaps, partly due to the constraint of using a bivariate power correlation function with a constant range and smoothness. Conversely, the estimates obtained with the bivariate extremal-Gaussian random field appear to overestimate the extremal dependence, because the extremal coefficient function under this model cannot exceed the upper bound, that is approximately 1.71 (Schlather, 2002).

Figure 5 shows 25 -year return levels for wind speed and gust. The location and scale marginal parameters at unobserved locations were estimated using quadratic regressions on longitude and latitude. The shape parameter was estimated assuming it is constant. We sampled 10,000 realizations from a residual bivariate extremal- $t$ random field with unit Fréchet marginals and with the dependence structure that is reported in Table 2. We used the realizations of the residual random 

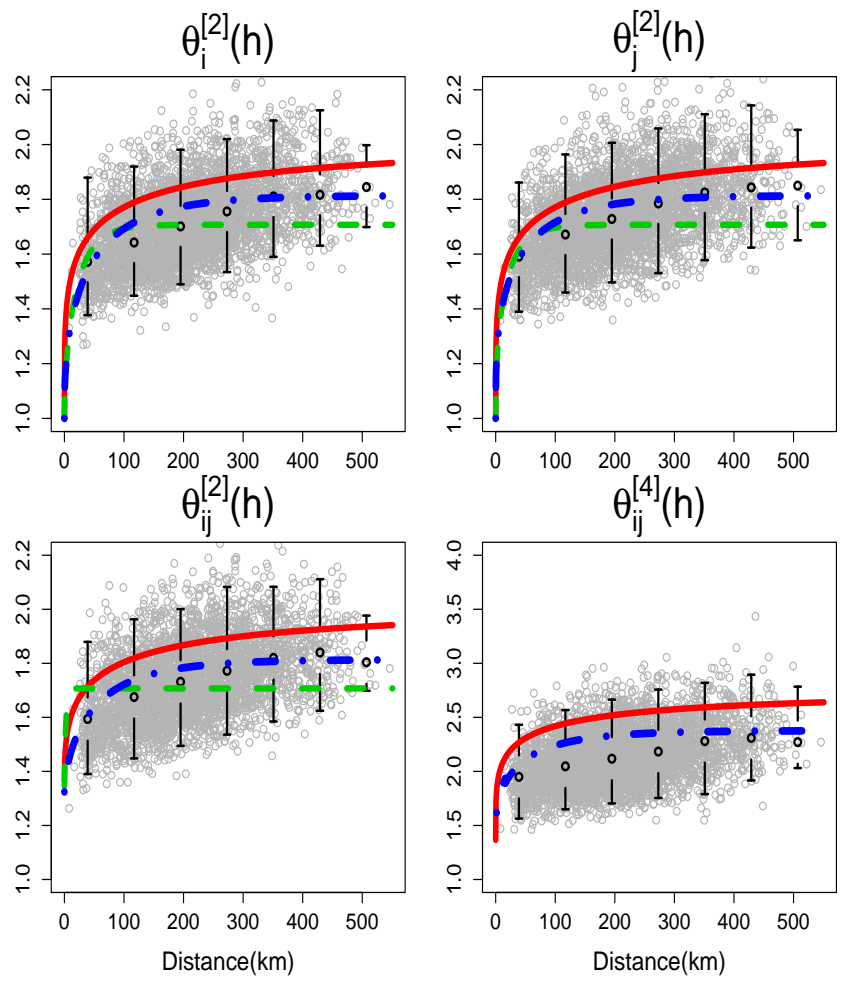

Fig. 4. Plots of the estimated inter- and cross-component extremal coefficient functions versus distance. The grey circles are the pairwise empirical extremal coefficients and the black circles are the binned empirical estimates. The vertical lines are the $95 \%$ confidence intervals. The curves are fitted extremal coefficient functions using the pairwise-likelihood under the bivariate Hüsler-Reiss (red solid line), extremal-Gaussian (green dashed line) and extremal- $t$ (blue dot-dashed line) random fields.

fields together with the marginal parameters to transform the data back to generalized extreme value marginals. The return levels were then estimated using the empirical quantiles of the sampled random fields. Figure 5 maps the 25-year conditional return levels of wind speed/gust given wind gust/speed at the same location or in Oklahoma City. The $T$-year conditional return level of wind speed/gust given wind gust/speed is defined as a threshold such that the conditional probability that wind speed/gust exceeds this threshold is $1 / T$, given that, at the same location, wind gust/speed exceeds its own $T$-year return level. The $T$-year conditional return level of wind speed/gust given the wind gust/speed in Oklahoma City is defined as a threshold such that the conditional probability that wind speed/gust exceeds this threshold is $1 / T$, given that the wind gust/speed in Oklahoma City exceeds its own $T$-year return level. The 25 -year conditional return levels of wind speed/gust given the wind gust/speed in Oklahoma City look similar to their unconditional counterparts, although they are greater by about 3.2 to 11.2 miles per hour, indicating that it is expected that a more extreme wind speed/gust event will be observed when an extreme wind gust/speed event has occurred in Oklahoma City. The 25-year conditional return levels of wind speed/gust given wind gust/speed are slightly higher than the 25-year conditional return levels of both wind speed/gust given wind gust/speed in Oklahoma City, indicating that 

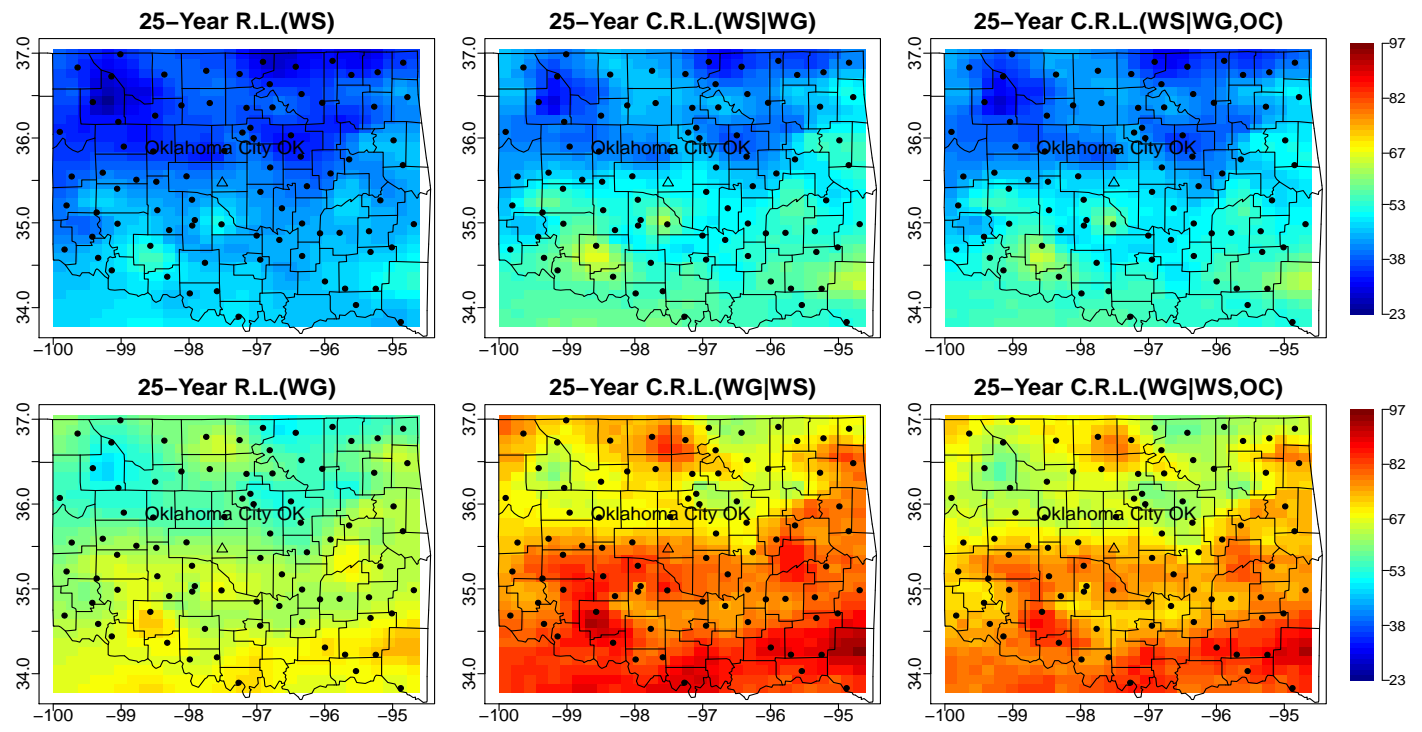

Fig. 5. Maps of the 25-year return levels for wind speed and gust (top-left and bottom-left column), maps of 25year conditional return levels for wind speed given gust and wind gust given speed (top-middle and bottom-middle column) and the 25-year conditional return levels for wind speed given wind gust in Oklahoma City and wind gust given wind speed in Oklahoma City (top-right and bottomright column) in miles per hour. Vertical and horizontal axes report latitude and longitude. The 83 weather stations are marked by black dots and Oklahoma City by a black triangle. Black lines delineate counties in Oklahoma.

observing a more extreme wind speed/gust event when an extreme wind gust/speed event has occurred at the same location is expected.

\section{ACKNOWLEDGMENT}

We are grateful to two anonymous referees, the associate editor and the editor for their comments that improved the exposition of the paper.

\section{SUPPLEMENTARY MATERIAL}

Supplementary material available at Biometrika online includes details for deriving the distributions of multivariate Hüsler-Reiss, extremal- $t$, Brown-Resnick and Gaussian extreme-value processes, the triplewise and quadruplewise Hüsler-Reiss distributions and simulation results for the trivariate Hüsler-Reiss process.

\section{REFERENCES}

Apanasovich, T. V. \& Genton, M. G. (2010). Cross-covariance functions for multivariate random fields based on latent dimensions. Biometrika 97, 15-30.

Apanasovich, T. V., Genton, M. G. \& Sun, Y. (2012). A valid Matérn class of cross-covariance functions for 390 multivariate random fields with any number of components. J. Am. Statist. Assoc. 107, 180-93. 
Brown, B. M. \& Resnick, S. I. (1977). A spectral representation for max-stable processes. J. Appl. Prob. 14, 732-9.

Cooley, D., NAveau, P. \& Poncet, P. (2006). Variograms for spatial max-stable random fields. In Ed. P. Bickel, P. Diggle, S. Fienberg, U. Gather, I. Olkin, S. Zeger, P. Bertail, P. Soulier and P. Doukhan, Dependence in Probability and Statistics, Lecture Notes in Statistics 187. Springer, pp. 373-90.

Cooley, D., Cisewski, J., Erhardt, R. J., Jeon, S., Mannshardt, E., Omolo B. O. \& Sun, Y. (2012). A survey of spatial extremes: Measuring spatial dependence and modeling spatial effects. REVSTAT Statist. J. 10, 135-65.

400 Davis, R. A., KlÜpperlberg, C. \& Steinkohl, C. (2013). Statistical inference for max-stable processes in space and time. J. R. Statist. Soc. B 75, 791-819.

Davison, A. C. \& Gholamrezaee, M. (2012). Geostatistics of extremes. P. Roy. Soc. A-Math. Phy. 468, 581-608.

Davison, A. C. \& Huser, R. (2014). Statistics of Extremes. Annual Rev. Statis. Appl. 2, in press.

Davison, A. C., Padoan, S. A. \& Ribatet, M. (2012). Statistical modeling of spatial extremes. Statist. Sci. 27, 161-86.

DE HAAN, L. (1984). A spectral representation for max-stable processes. Ann. Prob. 12, 1194-204.

DE HAAN, L. \& FerReIRA, A. (2006). Extreme Value Theory: An Introduction. New York: Springer.

DE HAAN, L. \& ZHOU, C. (2011). Extreme residual dependence for random vectors and processes. Adv. Appl. Prob. 43, 217-42.

Genton, M. G. \& Kleiber, W. (2015). Cross-covariance functions for multivariate geostatistics (with discussion). Statist. Sci., in press.

Genton, M. G., MA, Y. \& SANG, H. (2011). On the likelihood function of Gaussian max-stable processes. Biometrika 98, 481-8.

Gneiting, T., Kleiber, W. \& Schlather, M. (2010). Matérn cross-covariance functions for multivariate random fields. J. Am. Statist. Assoc. 105, 1167-77.

Huser, R. \& DAVISON, A. C. (2013). Composite likelihood estimation for the Brown-Resnick process. Biometrika $100,511-8$.

Huser, R. \& Davison, A. C. (2014). Space-time modelling of extreme events. J. R. Statist. Soc. B 76, 439-61.

HÜSLER, J. \& REISS, R.-D. (1989). Maxima of normal random vectors: Between independence and complete dependence. Statist. Prob. Lett. 7, 283-6.

Kabluchko, Z. L. (2009). Extremes of space-time Gaussian processes. Stoch. Proces. Appl. 119, 3962-80.

KABLUCHKO, Z. L. (2011). Extremes of independent Gaussian processes. Extremes 14, 285-310.

Kabluchko, Z., Schlather, M. \& DE HAan, L. (2009). Stationary max-stable fields associated to negative definite functions. Ann. Prob. 37, 2042-65.

425 Kleiber, W. \& Genton, M. G. (2013). Spatially varying cross-correlation coefficients in the presence of nugget effects. Biometrika 100, 213-20.

LI, B. \& ZHANG, H. (2011). An approach to modeling asymmetric multivariate spatial covariance structures. $J$. Mult. Anal. 102, 1445-53.

NikOloulopoulos, A. K., JoE, H. \& Li, H. (2009). Extreme value properties of multivariate $t$ copulas. Extremes 12, $129-48$.

OPITZ, T. (2013). Extremal t processes: Elliptical domain of attraction and a spectral representation. J. Mult. Anal. 122, 409-13.

Padoan, S. A., Ribatet, M. \& Sisson, S. A. (2010). Likelihood-based inference for max-stable processes. $J$. Am. Statist. Assoc. 105, 263-77.

435 Padoan, S. A. (2013a). Extreme dependence models based on event magnitude. J. Mult. Anal. 122, 1-19.

PadoAn, S. A. (2013b). Max-Stable Processes. Encyclopedia of Environmetrics 4, John Wiley \& Sons, Ltd, DOI: 10.1002/9780470057339.vnn022.

Resnick, S. I. (1987). Extreme Values, Point Processes and Regular Variation. New York: Springer Verlag, 1st Edition.

Ribatet, M., Cooley, D. \& Davison, A. C. (2012). Bayesian inference from composite likelihoods, with an application to spatial extremes. Statist. Sinica 22, 813-45.

RiBATET, M. (2013). Spatial extremes: Max-stable processes at work. J. Soc. Française Statist., 154, 156-77.

RøISLIEN, J. \& OMRE, H. (2006). T-distributed random fields: A parametric model for heavy-tailed well log data. Math. Geol. 38, 821-49.

SAnG, H. \& Genton, M. G. (2014). Tapered composite likelihood for spatial max-stable models. Spat. Statist. 8, 86-103.

SCHLATHER, M. (2002). Models for stationary max-stable random fields. Extremes 5, 33-44.

SChlather, M. \& TAWN, J. A. (2003). A dependence measure for multivariate and spatial extreme values: Properties and inference. Biometrika 90, 139-54.

450 VArin, C., ReID, N. \& FirTh, D. (2011). An overview of composite likelihood methods. Statist. Sinica 21, 5-42.

WADSWORTH, J. L. \& TAWn, J. A. (2012). Dependence modelling for spatial extremes. Biometrika 99, $253-72$.

WADsworth, J. L. \& TAWn, J. A. (2014). Efficient inference for spatial extreme value processes associated to log-Gaussian random functions. Biometrika 101, 1-15. 
Zhang, Z. \& SMith, R. L. (2004). The behavior of multivariate maxima of moving maxima processes. J. Appl. Prob. 41, 1113-23.

[Received April 2013. Revised September 2014] 